European Psychiatry

www.cambridge.org/epa

\title{
E-Poster Viewing
}

Anxiety disorders and somatoform disorders

\section{EPV0001}

Listening to music as a stress management tool

F. Lata and I. Kourtesis*

Department of Adult Psychiatry, Psychiatric Hospital of Athens "Dafni”, ATHENS, Greece

${ }^{*}$ Corresponding author.

doi: 10.1192/j.eurpsy.2021.1621

Introduction: The impact of listening to music on the stress response system has received increased attention lately.

Objectives: An update of the relative research.

Methods: Literature review.

Results: Listening to music seems to reduce stress by influencing both the hypothalamicpituitary-adrenal axis and the autonomous nervous system. Most studies, mainly conducted in healthy adults, show a decrease both in cortisol levels (a well-known stress-biomarker) and in sympathetic activity (reduction in heart rate frequency and blood pressure). Compositional elements of music such as melody, rhythm, tonality and frequency seem to influence individual relaxation responses. Most studies used classical music, nonetheless, the abovementioned effects were noticed irrespective of music genre. High-frequency music seems to have a greater role in stress-relief: $528 \mathrm{~Hz}$ frequency music apparently lowers cortisol and increases oxytocin (a modulator of stress response and social bonding) levels. A decrease in perceived anxiety and the induction of a positive mood state -particularly when relaxation was affirmed as the purpose of music listening- has been noticed using appropriate scales. Regarding particular settings, perioperative music may attenuate the neuroendocrine stress response caused by the surgery procedure. Music interventions in obstetric patients as well as in cardiovascular and cancer patients have led to similar findings. Finally, music appears to beneficially affect stress among patients with PSTD, fibromyalgia and depression.

Conclusions: The stress-relieving effect of music listening seems promising in clinical settings. The heterogeneity of the studies' sample and the "administration" of different music intervention "schemes" are among the main limitations of the current research.

Disclosure: No significant relationships.

Keywords: music listening; stress management

\section{EPV0002}

Monitoring anxiety symptoms with the monarca mobile application - an investigation of the usability during the first $\mathbf{1 8}$ months of commissioning in practice

K. Tarp ${ }^{1 \rtimes}$, T. Holmberg ${ }^{2}$, A.M. Møller ${ }^{3}$ and M. Lichtenstein ${ }^{4}$

${ }^{1}$ Research Unit For Telepsychiatry And E-mental Health, Centre For Telepsychiatry, Mental Health Services in the Region of Southern Denmark, Odense, Denmark; ${ }^{2}$ Research Unit For Telepsychiatry And Emental Health, Centre for Telepsychiatry, Odense C, Denmark; ${ }^{3}$ Health Promotion Research, Department Of Public Health, University of Southern Denmark, Odense, Denmark and ${ }^{4}$ Research Unit For Telepsychiatry And E-mental Health, Mental Health Services in the Region of Southern Denmark, Odense, Denmark

${ }^{*}$ Corresponding author. doi: 10.1192/j.eurpsy.2021.1622

Introduction: In cognitive behavioral treatment of anxiety disorders, registration of emotions and behavior is an important part of the intervention. Normally, paper and pencil is used but registrations on a mobile application such as MONARCA may be a useful alternative.

Objectives: This study investigates the usability of MONARCA during the first 18 months of commissioning in practice. 
Methods: To explore the usability, semi-structured interviews were conducted with seven patients and three therapists, combined with data from a survey questionnaire where 10 patients and 12 therapists rated the usability of the app on the System Usability Scale. Participants were recruited from an outpatient clinic for affective disorders in The Mental Health Services in the Region of Southern Denmark.

Results: Technical performance, time allocation, therapist effort, commitment, enthusiasm, and increased knowledge are imperative factors. Therapists and patients found that the benefits of registering emotions and behaviors on a mobile application were that it was easy for patients to remember to register daily, it was easy to gain an overview over symptom progress, and access to the registrations improved therapist's ability to prepare sessions.

Conclusions: Overall, the results from the interviews and survey indicated that both patients and therapists found MONARCA useful, but several improvement opportunities regarding application features and use in the treatment course were found.

Disclosure: No significant relationships.

Keywords: anxiety disorders; Anxiety monitoring; usability;

App-based assessment

\section{EPV0003}

\section{Severe depression when everything else is dismissed.} A case

L. Soldado Rodriguez ${ }^{1 \star}$, S.S. Sánchez Rus ${ }^{2}$ and A. Alvarado Dafonte ${ }^{2}$

${ }^{1}$ Mental Health Unit, Complejo Hospitalario de Jaen, Jaen, Spain and

${ }^{2}$ Jaén, Complejo Hospitalario Jaén, Jaén, Spain

${ }^{*}$ Corresponding author.

doi: 10.1192/j.eurpsy.2021.1623

Introduction: Simulation is a deliberate counterfeiting of physical or psychological symptoms in order to obtain a secondary gain or external incentive, like evading from military service, scape from work, obtain economic compensations or avoid criminal responsibility. It is estimated that prevalence is roughly $1 \%$ in mental health patients, with higher prevalence in young males and middle aged. Male with 52 years attends to emergency service. Erratic tracking in Mental Health Service from two years ago with unfavorable progress. He goes to emergency service referring aggravation of discomforted state of mind even with readjustment a week ago. Addiction to benzodiazepines and clinophilia. Currently with temporary inability to work of large data.

Objectives: To set a differential diagnose between depression, factitious disorder and malingering.

Methods: Examination shows moderated sad mood with despair, reactive to disability and progression of his illness. Sparing in words speech, focused on life or work problems. Autolytic verbalizations and self-control inability.

Results: Mmpi2 that shows: Gough's F-K. Dissimulation index, 34. Cut-off point to consider simulation/ pretending being ill varies among authors. A conservative cut-off point is 15, showing a severe exaggeration of its discomfort and dissimulation.

Conclusions: It is important to make an appropriate anamnesis and psychopathological exploration, as well as observation to reach a correct diagnose. In this case, clearly secondary gain was founded, therefore diagnose was malingering.

Disclosure: No significant relationships.

Keywords: Hospitalization; Simulation; factitius disorder; mmpi-2

\section{EPV0004}

\section{Mental illness pathogenia: Anxiety disorders, an evolutive vision}

L. Carpio Garcia ${ }^{\star}$, C. Martín Villarroel, M. Sánchez Revuelta, J. Matsuura, J. Dominguez Cutanda and E. García

Psychiatry, COMPLEJO HOSPITALARIO DE TOLEDO, Toledo, Spain

${ }^{*}$ Corresponding author.

doi: 10.1192/j.eurpsy.2021.1624

Introduction: There are many authors that follow and develop Pinel-hypotheses about unitary psychosis, joining recent discoveries in neuropathology and neurochemistry, supporting the vision of mental illness as neurodevelop disorders. The classification they suggest, distinguishes early, late neurodevelop disorders, and those related to traumatic factors, what determine an evolutive vision of this pathology. In terms of anxiety symptoms/disorders, they have been usually associated with categorical pathology, and treated focus on symptoms, unfortunately relapses are very frequent.

Objectives: Proving that the evolutive vision may ease a change on the intervention of anxiety disorders, that would propound different therapeutic alternatives.

Methods: A bibliographic search was performed from different databases, showing throw aspects related to main etiopathogenic theories about anxiety disorders from an evolutive vision.

Results: Evolutive-Psychology raises that anxiety is a concomitant process to development, that grows progressively and is necessary to induce changes in it. However a high level of anxiety might block that process or causes alterations. In that sense, anxiety-disorders may be related to an excess of anxiety that provoke a fault in present handling mechanisms. According to classic dynamic-theories, these mechanisms are associated with defence concept, but now we can link them to neurobiological development. From this point, there exists an asymmetric neurological maturation through childhood-adolescence that translates different manifestations of anxiety along development, initially more related with external contemption and relationship with caregiver, but later with hormonal pulses, physical changes and separation from family.

Conclusions: The evolutive vision allows to understand development fluctuation of anxiety symptoms along the growth process, more accurately than categorical classic tendency.

Disclosure: No significant relationships.

Keywords: Evolutive Psychology; Anxiety; neurobiological development 